\title{
Role of phosphodiesterase-4 inhibitors in chronic obstructive pulmonary disease
}

\author{
Chin Kook Rhee ${ }^{1}$ and Deog Kyeom Kim ${ }^{2}$
}

\author{
${ }^{1}$ Division of Pulmonary, Allergy \\ and Critical Care Medicine, \\ Department of Internal Medicine, \\ Seoul St. Mary's Hospital, College of \\ Medicine, The Catholic University of \\ Korea, Seoul; ${ }^{2}$ Division of Pulmonary \\ and Critical Care Medicine, \\ Department of Internal Medicine, \\ Seoul Metropolitan Government \\ Seoul National University Boramae \\ Medical Center, Seoul, Korea
}

Phosphodiesterase-4 inhibitors (PDE4Is) are potent anti-inflammatory agents and roflumilast has been used to prevent acute exacerbation of chronic obstructive pulmonary disease (COPD). Roflumilast decreases neutrophil migration, restores cystic fibrosis transmembrane conductance regulator activity, and recovers glucocorticoid effects. A forced expiratory volume in 1 second of $<50 \%$, a chronic bronchitis phenotype, high blood eosinophil levels, and a history of hospitalization are biomarkers for predicting responses to roflumilast. Adverse effects are common in clinical practice. An inhaled $\mathrm{PDE}_{4} \mathrm{I}$ has recently been developed and is under clinical trial. CHF60o1 and RPL554 exhibit promise and may be future treatment options for COPD.

Keywords: Pulmonary disease, chronic obstructive; Phosphodiesterase 4 inhibitors; Roflumilast

\section{INTRODUCTION}

Chronic obstructive pulmonary disease (COPD) is a leading cause of death worldwide $[1,2]$. Inhaled medications are currently standard for COPD treatment. Other oral medications such as phosphodiesterase-4 inhibitors (PDE4Is) can be added for treating a subgroup of COPD, based on specific criteria. As inflammation is a key component in the pathogenesis of COPD, $\mathrm{PDE}_{4}$ Is are expected to be effective in suppressing inflammation in COPD. In addition, they are clinically proven to prevent exacerbation in severe COPD patients. A review of the potential beneficial mechanisms of PDE4 Is is important and can be helpful to clinicians for managing COPD patients. In the future, a new-generation $\mathrm{PDE}_{4} \mathrm{I}$ in an inhaled form may be available. Therefore, an understanding of the strengths and weaknesses of PDE4Is can guide physicians to better manage COPD patients.

\section{PDE4I MECHANISM OF ACTION IN AIRWAY DISEASES}

COPD is associated with increased inflammation in airways. Previous studies have showed that PDE4Is can suppress the activities of many pro-inflammatory and immune cells in COPD [3]. Roflumilast is a targeted oral once-daily $\mathrm{PDE}_{4} \mathrm{I}$ that is currently available in practice. Grootendorst et al. [4] showed that roflumilast treatment reduced the number of neutrophils and eosinophils in induced sputum.

Diseases. 
Also, the levels of soluble interleukin-8, neutrophil elastase, eosinophil cationic protein, and $\alpha 2$-macroglobulin were significantly reduced with roflumilast.

Although the detailed mechanism of how roflumilast suppresses inflammation in COPD patients is not well-understood, the self-propagating acetyl-proline-glycine-proline (AcPGP) pathway is involved in neutrophilic inflammation in COPD. Wells et al. [5] conducted a placebo-controlled randomized study and found that 12 weeks of roflumilast treatment significantly decreased sputum AcPGP by $>50 \%$. Roflumilast reduces pulmonary inflammation via lowering prolyl endopeptidase activity.

The anti-inflammatory effect of roflumilast on neutrophils may occur through an increase in cyclic adenosine monophosphate (cAMP) and the suppression of cytokine [6]. Alternatively, roflumilast can act directly on neutrophils to suppress their function. Roflumilast prevented endothelial P- and E-selectin expression in vivo. In vitro, roflumilast $\mathrm{N}$-oxide (RNO) suppressed neutrophil adhesion to endothelial cells [7]. Recently, Dunne et al. [8] reported that roflumilast had an inhibitory effect on neutrophil migration. Neutrophils in COPD patients displayed increased chemotaxis, which was inhibited by RNO in a dose-dependent manner.

Cystic fibrosis transmembrane conductance regulator (CFTR) is cAMP-regulated anion transporter. It is expressed on the apical surface of epithelial cells in the lung. Cigarette smoking can cause CFT'R dysfunction, which results in delayed mucus clearance and chronic bronchitis. An imbalance of ion transport combined with mucus hypersecretion increases the mucin concentration in airways [9]. This can lead to bacterial colonization and the progression of COPD. Restoration of CFTR activity is an ideal way of managing chronic bronchitis and further preventing COPD progression (Fig. 1). Lambert et al. [10] showed that roflumilast activates CFTR and mitigates CFTR dysfunction caused by cigarette smoking. Interestingly, roflumilast also activates CFTR in the intestinal epithelium. This could be a plausible mechanism for the diarrhea that is a common adverse effect of roflumilast. Schmid et al. [11] also showed that roflumilast can rescue smoke-induced mucociliary dysfunction by reversing the decrease in

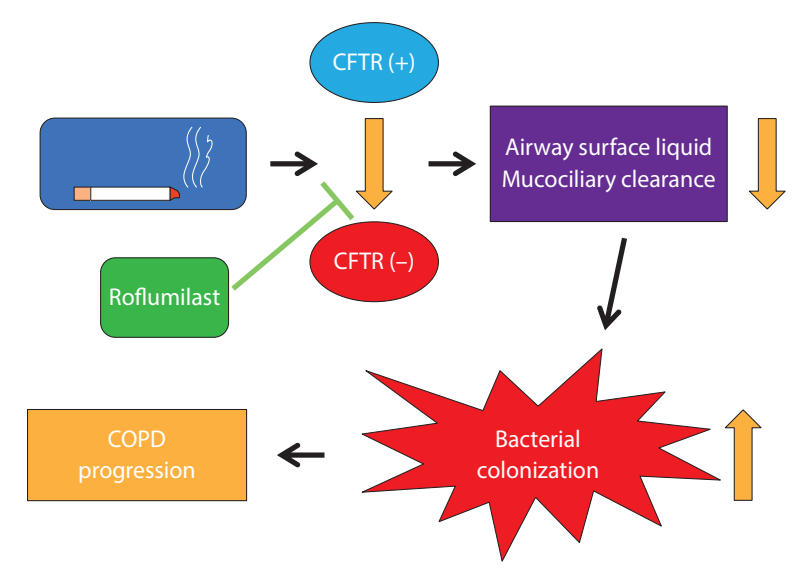

Figure 1. Beneficial effects of roflumilast in chronic bronchitis via the activation of the cystic fibrosis transmembrane conductance regulator (CFTR). COPD, chronic obstructive pulmonary disease.

CFTR activity. Moreover, PDE4 inhibition decreased mucin $5 \mathrm{AC}$ (MUC5 $\mathrm{AC}$ ) expression in human airway epithelial cells [12]. These effects of PDE4Is can help with decreasing mucus secretion and increasing mucus clearance.

Steroid resistance is also an important issue in managing COPD. Glucocorticoids are effective anti-inflammatory drugs for treating asthma but are relatively ineffective in COPD. Reduced histone deacetylase 2 (HDAC2) expression and activity, impaired corticosteroid activation of the glucocorticoid receptor, and increased pro-inflammatory signaling pathways have been suggested as mechanisms of steroid resistance [13]. Recovering the anti-inflammatory effect of glucocorticoids is an ideal way to manage COPD. Milara et al. [14] isolated peripheral blood neutrophils from COPD patients and incubated them with RNO. Neutrophils from COPD stimulated with toll-like receptor 3 agonists exhibited corticosteroid resistance with a decrease in HDAC activity. RNO reversed the corticosteroid resistance, leading to robust anti-inflammatory effects, especially when combined with dexamethasone. These outcomes have also been observed in clinical trials. In a post hoc analysis of M2-111 and -112 trials, patients who received inhaled corticosteroids (ICS) exhibited better responses to roflumilast [15]. These results suggest that roflumilast can be effective in overcoming corticosteroid resistance in $\mathrm{COPD}$, via restoration of $\mathrm{HDAC} 2$ activity. 


\section{BIOMARKERS FOR PREDICTING THE RESPONSE TO ROFLUMILAST}

A subgroup analysis of an early phase III trial revealed that roflumilast is effective in decreasing COPD exacerbation in only a limited population [15]. The target population was confirmed in a subsequent trial with narrow enrollment criteria [16]. A forced expiratory volume in 1 second $\left(\mathrm{FEV}_{1}\right)$ of $<50 \%$, a chronic bronchitis phenotype, and a history of exacerbation are well-known biomarkers for predicting the response to roflumilast.

Another potential biomarker is high blood eosinophil levels. In a pooled analysis, Martinez et al. [17] reported that the decrease in exacerbation by roflumilast was more pronounced in patients with high blood eosinophil levels. In patients with a history of hospitalization, roflumilast reduced the exacerbation level by $34.5 \%$ at $\geq$ 150 cells $/ \mu \mathrm{L}$ and $42.7 \%$ at $\geq 300$ cells $/ \mu$ l compared with a placebo. Rabe et al. [18] aimed to assess the anti-inflammatory effects of roflumilast on bronchial mucosa. Significant reductions in eosinophil cell counts in bronchial biopsy samples and induced sputum after 16 weeks of roflumilast treatment were noted. This result is compatible with the findings from a chronic asthma model, in which administration of roflumilast significantly decreased eosinophilic inflammation [19]. Considering that roflumilast was initially developed as an asthma medication, it is not surprising that roflumilast can decrease eosinophilic inflammation.

A history of exacerbation is a well-known biomarker for predicting the response to roflumilast. However, recent studies suggested that a history of hospital admission can be a more powerful predictor. In a 52week phase 4 trial, roflumilast failed to decrease the exacerbation level compared with a placebo in patients administered an ICS/long-acting beta2 agonist with or without a long-acting muscarinic antagonist [20]. However, a post hoc analysis found that roflumilast significantly reduced exacerbation levels in patients with a history of more than three exacerbation events and/or one or more hospitalizations. Another post hoc analysis also showed that roflumilast is more effective in patients with a history of hospitalization [21]. Roflumilast reduced severe exacerbation by $35 \%$ in patients with a prior hospitalization $(p=0.007)$ but by only $8 \%$ in patients without such a history $(p=0.634)$.

\section{EFFECTS OF ROFLUMILAST ON COMORBIDITIES}

COPD is a chronic inflammatory disease, and inflammation in COPD is not limited to the lung. Increased systemic inflammation is also an important feature of COPD. Agusti et al. [22] showed that systemic inflammation is significantly elevated in COPD, and that persistent systemic inflammation was associated with poor clinical outcomes. It is well-known that COPD patients have comorbidities associated with this elevated systemic inflammation [23]. It is difficult for inhaled medication to decrease systemic inflammation in COPD, as systemic absorption of inhaled medication is expected to be low. However, roflumilast is an oral medication and is systemically absorbed. Thus, the anti-inflammatory effect of roflumilast may not be confined to lungs. Although there is limited evidence that roflumilast decreases systemic inflammation directly, there is increasing evidence that roflumilast can affect comorbid diseases.

Cardiovascular diseases are common and serious comorbid conditions in COPD. Placebo-controlled clinical trials of roflumilast were conducted to assess the rates of major adverse cardiovascular events (MACEs) [24]. The MACE rate was significantly lower in cases treated with roflumilast compared with those treated with a placebo (hazard ratio, 0.65 ). This potential benefit needs to be evaluated in prospective clinical trials.

In animal experiments, the $\mathrm{PDE}_{4} \mathrm{I}$ rolipram increased plasma glucagon-like peptide-1 (GLP-1) concentration and enhanced GLP-1 release [25]. GLP-1 receptor agonists are currently used for the treatment of diabetes. Wouters et al. [26] conducted a 12-week randomized multicenter study on the effects of roflumilast on glucose metabolism in patients with diabetes mellitus. Roflumilast increased insulin sensitivity and was associated with a significantly greater reduction in glycated hemoglobin [27].

\section{ADVERSE EFFECTS}

Perhaps the biggest barrier to the use of roflumilast in clinical practice is its adverse effects. Diarrhea, weight loss, nausea, headache, insomnia, decreased appetite, and 
dizziness are common. [28]. It is recommended that the development of adverse effects should be predicted before roflumilast is prescribed. Kim et al. [29] reported that patients with a low body mass index (BMI) were more likely to discontinue roflumilast. The odds ratio (OR) for discontinuation in patients with $\mathrm{BMI}<23\left(\mathrm{~kg} / \mathrm{m}^{2}\right)$ was 2.96. Over $40 \%$ of patients with BMI $<23$ could not tolerate roflumilast. It is important to reduce the side effects of roflumilast in clinical practice. A lower dose $(250 \mu \mathrm{g})$ may prevent adverse effects. Joo et al. [30] reported that the incidence of adverse effects was $38.2 \%$ with a 500- $\mu$ g dose and $25.3 \%$ with $250 \mu \mathrm{g}(p=0.034)$. The discontinuation rate was $41.6 \%$ with $500 \mu \mathrm{g}$ and $23.1 \%$ with $250 \mu \mathrm{g}(p=0.003)$. In multivariate analysis, the OR for discontinuation with $500 \mu \mathrm{g}$ was 2.87 . Similar results were reported in a multicenter, double-blind trial [31]. COPD patients ( $\mathrm{n}=1,321$ ) were randomly assigned to groups treated with roflumilast $250 \mu \mathrm{g}$ once daily, 500 $\mu \mathrm{g}$ every other day, or $500 \mu \mathrm{g}$ once daily for 4 weeks. Significantly fewer patients treated with $250 \mu \mathrm{g}$ once daily discontinued treatment (OR, o.66) and they had fewer side effects (OR, o.63) compared with the patients treated with $500 \mu \mathrm{g}$ once daily.

\section{USE OF ROFLUMILAST DURING EXACERBATION}

Most clinical trials of roflumilast aimed to show decreased exacerbation in patients with COPD. Enrolled patients should be stable (not undergoing exacerbation), although they may have a history of exacerbation in the past. Only one study examined the effect of roflumilast during an exacerbation event. Mackay et al. [32] performed a randomized, double-blind, placebo-controlled study to examine the effect of roflumilast addon during an exacerbation event. All patients received standardized exacerbation treatment. Compared with group receiving the placebo, numerically larger changes in $\mathrm{FEV}_{1}$ were observed in the roflumilast group. Roflumilast significantly decreased airway inflammation compared with the placebo. The percentage of sputum neutrophils $(-13.9 \%, p=0.049)$ and myeloperoxidase levels $(-7,800.6 \mathrm{ng} / \mathrm{mL}, p=0.018)$ decreased significantly. However, adverse events such as diarrhea $(65 \%$ with roflumilast vs. $23.3 \%$ with the placebo) and insomnia
(13.6\% vs. $4.7 \%$, respectively) were commonly reported. Side effects led to a withdrawal rate of $26.3 \%$ in the roflumilast group and $4.7 \%$ in the placebo group.

\section{INHALED PDE4ls}

Systemic administration of PDE4Is can cause side effects including gastrointestinal tract disturbance. To overcome this limitation, an inhaled PDE4I has been developed. CHF6oor is formulated to be delivered via inhalation. Recently, the results of a phase IIa study were reported by Singh et al. [33]. CHF6oo1 was well tolerated, and there was no difference in the incidence of gastrointestinal adverse events when CHF6oor or a placebo was used. Compared with the placebo, CHF6oor significantly decreased levels of leukotriene-B4 and -C, C-X-C motif chemokine ligand 8, macrophage inflammatory protein $1 \beta$, matrix metalloproteinase 9 , and tumor necrosis factor- $\alpha$ (TNF- $\alpha$ ) in sputum. Serum surfactant protein D levels also decreased significantly in the CHF6oor group. Interestingly, enrolled patients were already treated using triple inhaled therapy.

CHF6oor is seven-fold more potent than roflumilast in inhibiting PDE4 enzymatic activity [34]. It is designed for inhaled administration and characterized by high lung retention and low plasma levels. Lea et al. [35] isolated alveolar macrophages from COPD patients. CHF6001 significantly reduced TNF- $\alpha$ production in alveolar macrophages and lung tissue. It also inhibited the secretion of CCL-2 and -4 from macrophages.

RPL554 is a first-in-class, dual inhibitor of PDE3 and 4. $\mathrm{PDE}_{3}$ inhibition resulted in bronchodilation [36]. Thus, a dual inhibitor has an advantage compared with PDE4Is. A short-term bronchodilator effect of RPL554 was reported by Singh et al. [37]. Compared with ipratropium, the peak $\mathrm{FEV}_{1}$ was higher in cases treated with RPL554 and ipratropium (mean difference, $94 \mathrm{~mL} ; p<0.0001$ ). Using salbutamol as an addon also significantly increased the $\mathrm{FEV}_{1}$ by $108 \mathrm{~mL}$ $(p<0.0001)$. Compared with the placebo, the average $\mathrm{FEV}_{1(\mathrm{o}-12 \mathrm{~h})}$ increase was greater (mean difference, 65 $\mathrm{mL} ; p=0.0009)$. RPL554 was also well-tolerated alone or in combination with a short-acting bronchodilator. There was no significant difference in the incidence of adverse events between groups. 
Table 1. Suggested topics for future research on phosphodiesterase-4 inhibitors

\begin{tabular}{|c|c|c|c|}
\hline $\mathrm{PDE}_{4} \mathrm{I}$ & Topic & Subject & Main outcome \\
\hline \multirow[t]{4}{*}{ Roflumilast } & Comparison between 250 and $500 \mu \mathrm{g}$ & $\begin{array}{l}\text { Severe or very severe COPD with history } \\
\text { of frequent exacerbation and } \\
\text { chronic bronchitis }\end{array}$ & $\begin{array}{l}\text { Exacerbation } \\
\text { Adverse effect }\end{array}$ \\
\hline & Treatment of acute exacerbation & Patients with severe exacerbation state & $\begin{array}{l}\text { Mortality } \\
\text { ICU admission } \\
\text { Treatment failure } \\
\text { Length of hospitalization }\end{array}$ \\
\hline & Disease modifying effect & $\begin{array}{l}\text { Early COPD } \\
\text { Pre COPD }\end{array}$ & $\begin{array}{l}\mathrm{FEV}_{1} \text { decline } \\
\text { Progression of functional } \\
\text { small airway disease }\end{array}$ \\
\hline & Role as a mucoactive drug & $\begin{array}{l}\text { Early COPD } \\
\text { Pre COPD }\end{array}$ & Chronic bronchitis symptom \\
\hline \multirow[t]{2}{*}{ CHF6001 } & Phase III study & GOLD D & Exacerbation \\
\hline & Role as a mucoactive drug & $\begin{array}{l}\text { Early COPD } \\
\text { Pre COPD }\end{array}$ & Chronic bronchitis symptom \\
\hline \multirow[t]{3}{*}{ RPL554 } & Phase III study & GOLD D & Exacerbation \\
\hline & Effect beyond roflumilast & GOLD B & $\begin{array}{l}\mathrm{FEV}_{1} \\
\text { SGRQ } \\
\text { TDI }\end{array}$ \\
\hline & Role as a mucoactive drug & $\begin{array}{l}\text { Early COPD } \\
\text { Pre COPD }\end{array}$ & Chronic bronchitis symptom \\
\hline
\end{tabular}

PDE4I, phosphodiesterase 4 inhibitor; COPD, chronic obstructive pulmonary disease; ICU, intensive care unit; GOLD, the Global Initiative for Chronic Obstructive Lung Disease; $\mathrm{FEV}_{1}$, forced expiratory volume in 1 second; SGRQ, St. George's Respiratory Questionnaire; TDI, transition dyspnea index.

\section{FUTURE RESEARCH DIRECTIONS}

Several issues need to be validated in future research (Table 1). First, there are not enough data regarding the role of a $250-\mu \mathrm{g}$ dose of roflumilast. Although $250 \mu \mathrm{g}$ of roflumilast did not lead to a decrease in exacerbation in a previous clinical trial [38], it significantly improved lung function. Enrollment criteria in that study did not match the current indications for roflumilast. No Asian patients participated in this $250-\mu \mathrm{g}$ trial, although the BMI of Asian COPD patients is usually much lower [39-41]. The pharmacokinetics of this drug are related to BMI. Thus, it is difficult to generalize the results of this previous study using $250 \mu \mathrm{g}$ of roflumilast to an Asian population. For example, there was no significant difference in severe exacerbation rate between the 250and 500- $\mu \mathrm{g}$ groups [30]. The percentage of patients who experienced severe exacerbation while taking $500 \mu \mathrm{g}$ of roflumilast was $18.1 \%$, whereas that of patients taking a $250-\mu$ g dose was $14.3 \%$. Further clinical trials that com- pare efficacy and safety between doses of $250 \mu \mathrm{g}$ and 500 $\mu \mathrm{g}$ are necessary.

Second, the role of roflumilast is currently limited to the prevention of exacerbation. However, this needs to be extended to the treatment of exacerbation. For several decades, only two medications, systemic steroids and antibiotics, have been used for the treatment of acute exacerbation. Roflumilast has a potent anti-inflammatory effect. Thus, it is reasonable to try roflumilast during an exacerbation event. Because only one previous clinical trial produced positive results [32], a larger clinical trial should be performed in the future. Adverse effects were a major barrier in previous clinical trials. Thus, a $250-\mu \mathrm{g}$ dose rather than a 500- $\mu \mathrm{g}$ dose should be considered in future trials.

Third, the role of $\mathrm{PDE}_{4} \mathrm{Is}$ should be investigated in early COPD patients. No medication has been shown to slow disease progression. As COPD is a chronic inflammatory disease and PDE4Is are potent anti-inflammatory agents, a clinical trial on disease modification with 


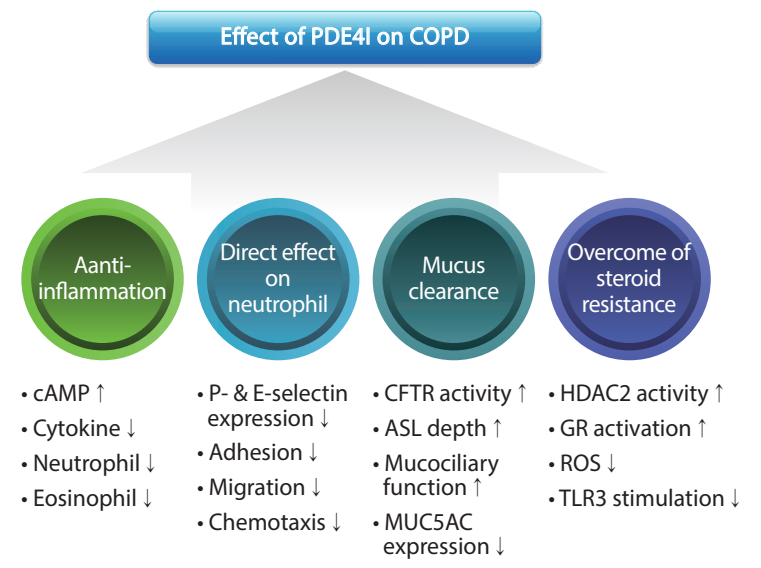

Figure 2. Mechanism of action of phosphodiesterase-4 inhibitors (PDE4Is) in chronic obstructive pulmonary disease (COPD). cAMP, cyclic adenosine monophosphate; CFTR, cystic fibrosis transmembrane conductance regulator; ASL, airway-surface liquid; MUC5AC, mucin 5AC; HDAC2, histone deacetylase 2; GR, glucocorticoid receptor; ROS, reactive oxygen species; $\mathrm{TLR}_{3}$, toll-like receptor 3 .

early COPD patients as targets would be worthwhile. The primary positive outcome could be a slower decline in $\mathrm{FEV}_{1}$; however, functional small airway disease could be an alternative target.

Fourth, the role of roflumilast as a mucoactive drug should be evaluated. Until now, all clinical trials of roflumilast were focused on exacerbation. Considering that roflumilast is effective in treating chronic bronchitis patients with CFTR activation as one of the mechanisms, it is reasonable to examine the effects of roflumilast on chronic bronchitis symptoms.

Fifth, as an inhaled PDE4I has been developed, a large phase III clinical trial should be performed. Whether inhaled $\mathrm{PDE}_{4} \mathrm{I}$ should be limited to patients with severe symptoms and a history of frequent exacerbation is also in question. The effects of the inhaled PDE4I on not only exacerbation but also lung function and chronic bronchitis symptoms need to be examined.

\section{CONCLUSIONS}

PDE4Is are potent anti-inflammatory agents that have been used to prevent COPD exacerbation. Other mechanisms such as CFTR activation and overcoming corticosteroid resistance are being evaluated and elucidated (Fig. 2). High blood eosinophil levels and a history of hospitalization are emerging new biomarkers that better predict the outcomes of roflumilast along with the classic biomarkers of $\mathrm{FEV}_{1}<50 \%$ and a chronic bronchitis phenotype. Adverse effects are common in clinical practice, and a $250-\mu \mathrm{g}$ dose may reduce the incidence of such effects. A recently developed inhaled $\mathrm{PDE}_{4} \mathrm{I}$ exhibits promise and is currently being assessed in ongoing clinical trials. Future $\mathrm{PDE}_{4} \mathrm{I}$ research should be focused on disease modification.

\section{Conflict of interest}

No potential conflict of interest relevant to this article was reported.

\section{REFERENCES}

1. Kim C, Kim Y, Yang DW, et al. Direct and indirect costs of chronic obstructive pulmonary disease in Korea. Tuberc Respir Dis (Seoul) 2019;82:27-34.

2. Rhee CK. Old friends, old wine, and old COPD classification are best. Tuberc Respir Dis (Seoul) 2018;81:347-348.

3. Giembycz MA. Development status of second generation $\mathrm{PDE}_{4}$ inhibitors for asthma and COPD: the story so far. Monaldi Arch Chest Dis 2002;57:48-64.

4. Grootendorst DC, Gauw SA, Verhoosel RM, et al. Reduction in sputum neutrophil and eosinophil numbers by the PDE4 inhibitor roflumilast in patients with COPD. Thorax 2007;62:1081-1087.

5. Wells JM, Jackson PL, Viera L, et al. A randomized, placebo-controlled trial of roflumilast: effect on proline-glycine-proline and neutrophilic inflammation in chronic obstructive pulmonary disease. Am J Respir Crit Care Med 2015;192:934-942.

6. Giembycz MA, Newton R. How phosphodiesterase 4 inhibitors work in patients with chronic obstructive pulmonary disease of the severe, bronchitic, frequent exacerbator phenotype. Clin Chest Med 2014;35:203-217.

7. Sanz MJ, Cortijo J, Taha MA, et al. Roflumilast inhibits leukocyte-endothelial cell interactions, expression of adhesion molecules and microvascular permeability. $\mathrm{Br} \mathrm{J}$ Pharmacol 2007;152:481-492.

8. Dunne AE, Kawamatawong T, Fenwick PS, et al. Direct inhibitory effect of the $\mathrm{PDE}_{4}$ inhibitor roflumilast on neutrophil migration in chronic obstructive pulmonary disease. Am J Respir Cell Mol Biol 2019;60:445-453. 
9. Kesimer M, Ford AA, Ceppe A, et al. Airway mucin concentration as a marker of chronic bronchitis. N Engl J Med 2017;377:911-922.

10. Lambert JA, Raju SV, Tang LP, et al. Cystic fibrosis transmembrane conductance regulator activation by roflumilast contributes to therapeutic benefit in chronic bronchitis. Am J Respir Cell Mol Biol 2014;50:549-558.

11. Schmid A, Baumlin N, Ivonnet P, et al. Roflumilast partially reverses smoke-induced mucociliary dysfunction. Respir Res 2015;16:135.

12. Mata M, Sarria B, Buenestado A, Cortijo J, Cerda M, Morcillo EJ. Phosphodiesterase 4 inhibition decreases MUC5AC expression induced by epidermal growth factor in human airway epithelial cells. Thorax 2005;60:144-152.

13. Barnes PJ. Mechanisms and resistance in glucocorticoid control of inflammation. J Steroid Biochem Mol Biol 2010;120:76-85.

14. Milara J, Lluch J, Almudever P, Freire J, Xiaozhong Q, Cortijo J. Roflumilast N-oxide reverses corticosteroid resistance in neutrophils from patients with chronic obstructive pulmonary disease. J Allergy Clin Immunol 2014;134:314-322.

15. Rennard SI, Calverley PM, Goehring UM, Bredenbroker D, Martinez FJ. Reduction of exacerbations by the PDE4 inhibitor roflumilast: the importance of defining different subsets of patients with COPD. Respir Res 2011;12:18.

16. Calverley PM, Rabe KF, Goehring UM, et al. Roflumilast in symptomatic chronic obstructive pulmonary disease: two randomised clinical trials. Lancet 2009;374:685-694.

17. Martinez FJ, Rabe KF, Calverley PM, et al. Determinants of response to roflumilast in severe chronic obstructive pulmonary disease: pooled analysis of two randomized trials. Am J Respir Crit Care Med 2018;198:1268-1278.

18. Rabe KF, Watz H, Baraldo S, et al. Anti-inflammatory effects of roflumilast in chronic obstructive pulmonary disease (ROBERT): a 16-week, randomised, placebo-controlled trial. Lancet Respir Med 2018;6:827-836.

19. Kim SW, Kim JH, Park CK, et al. Effect of roflumilast on airway remodelling in a murine model of chronic asthma. Clin Exp Allergy 2016;46:754-763.

20. Martinez FJ, Rabe KF, Sethi S, et al. Effect of roflumilast and inhaled corticosteroid/long-acting $\beta 2$-agonist on chronic obstructive pulmonary disease exacerbations (RE(2)SPOND): a randomized clinical trial. Am J Respir Crit Care Med 2016;194:559-567.

21. Rabe KF, Calverley PM, Martinez FJ, Fabbri LM. Effect of roflumilast in patients with severe COPD and a history of hospitalisation. Eur Respir J 2017;50:1700158.

22. Agusti A, Edwards LD, Rennard SI, et al. Persistent systemic inflammation is associated with poor clinical outcomes in COPD: a novel phenotype. PLoS One 2012;7:e37483.

23. Fabbri LM, Rabe KF. From COPD to chronic systemic inflammatory syndrome? Lancet 2007;370:797-799.

24. White WB, Cooke GE, Kowey PR, et al. Cardiovascular safety in patients receiving roflumilast for the treatment of COPD. Chest 2013;144:758-765.

25. Ong WK, Gribble FM, Reimann F, et al. The role of the PDE4D cAMP phosphodiesterase in the regulation of glucagon-like peptide-1 release. Br J Pharmacol 2009;157:633-644.

26. Wouters EF, Bredenbroker D, Teichmann P, et al. Effect of the phosphodiesterase 4 inhibitor roflumilast on glucose metabolism in patients with treatment-naive, newly diagnosed type 2 diabetes mellitus. J Clin Endocrinol Metab 2012;97:E1720-E1725.

27. Wedzicha JA, Calverley PM, Rabe KF. Roflumilast: a review of its use in the treatment of COPD. Int J Chron Obstruct Pulmon Dis 2016;11:81-90.

28. Garnock-Jones KP. Roflumilast: a review in COPD. Drugs 2015;75:1645-1656.

29. Kim KH, Kang HS, Kim JS, Yoon HK, Kim SK, Rhee CK. Risk factors for the discontinuation of roflumilast in patients with chronic obstructive pulmonary disease. Int J Chron Obstruct Pulmon Dis 2017;12:3449-3456.

30. Joo H, Han D, Lee JH, Rhee CK. Incidence of adverse effects and discontinuation rate between patients receiving 250 micrograms and 500 micrograms of roflumilast: a comparative study. Tuberc Respir Dis (Seoul) 2018;81:299304.

31. Watz H, Bagul N, Rabe KF, et al. Use of a 4-week up-titration regimen of roflumilast in patients with severe COPD. Int J Chron Obstruct Pulmon Dis 2018;13:813-822.

32. Mackay AJ, Patel AR, Singh R, et al. Randomized double-blind controlled trial of roflumilast at acute exacerbations of chronic obstructive pulmonary disease. Am J Respir Crit Care Med 2017;196:656-659.

33. Singh D, Beeh KM, Colgan B, et al. Effect of the inhaled $\mathrm{PDE}_{4}$ inhibitor CHF6oor on biomarkers of inflammation in COPD. Respir Res 2019;20:180.

34. Moretto N, Caruso P, Bosco R, et al. CHF6oor I: a novel highly potent and selective phosphodiesterase 4 inhibitor 
with robust anti-inflammatory activity and suitable for topical pulmonary administration. J Pharmacol Exp Ther 2015;352:559-567.

35. Lea S, Metryka A, Li J, et al. The modulatory effects of the $\mathrm{PDE}_{4}$ inhibitors $\mathrm{CHF} 6001$ and roflumilast in alveolar macrophages and lung tissue from COPD patients. Cytokine 2019;123:154739.

36. Myou S, Fujimura M, Kamio Y, et al. Bronchodilator effect of inhaled olprinone, a phosphodiesterase 3 inhibitor, in asthmatic patients. Am J Respir Crit Care Med 1999;160:817-820.

37. Singh D, Abbott-Banner K, Bengtsson T, Newman K. The short-term bronchodilator effects of the dual phosphodiesterase 3 and 4 inhibitor RPL554 in COPD. Eur Respir J 2018;52:1801074.

38. Rabe KF, Bateman ED, O’Donnell D, Witte S, Bredenbro- ker D, Bethke TD. Roflumilast: an oral anti-inflammatory treatment for chronic obstructive pulmonary disease: a randomised controlled trial. Lancet 2005;366:563-571.

39. Lim JU, Lee JH, Kim JS, et al. Comparison of World Health Organization and Asia-Pacific body mass index classifications in COPD patients. Int J Chron Obstruct Pulmon Dis 2017;12:2465-2475.

40. Jo YS, Kim YH, Lee JY, et al. Impact of BMI on exacerbation and medical care expenses in subjects with mild to moderate airflow obstruction. Int J Chron Obstruct Pulmon Dis 2018;13:2261-2269.

41. Rhee CK, Chau NQ, Yunus F, Matsunaga K, Perng DW; on behalf the COPD Assembly of the APSR. Management of COPD in Asia: a position statement of the Asian Pacific Society of Respirology. Respirology 2019;24:1018-1025. 